\title{
Dirichlet Boundary Value Problem for the Second Order Asymptotically Linear System
}

\author{
A. Gritsans, ${ }^{1}$ F. Sadyrbaev, ${ }^{1,2}$ and I. Yermachenko ${ }^{1}$ \\ ${ }^{1}$ Institute of Life Sciences and Technologies, Daugavpils University, Parades iela $1^{a}$, Daugavpils LV-5400, Latvia \\ ${ }^{2}$ Institute of Mathematics and Computer Science, University of Latvia, Raina bulv. 29, Riga LV-1459, Latvia \\ Correspondence should be addressed to A. Gritsans; arminge@inbox.lv
}

Received 27 July 2016; Accepted 5 October 2016

Academic Editor: Qingkai Kong

Copyright (c) 2016 A. Gritsans et al. This is an open access article distributed under the Creative Commons Attribution License, which permits unrestricted use, distribution, and reproduction in any medium, provided the original work is properly cited.

We consider the second order system $\mathbf{x}^{\prime \prime}=\mathbf{f}(\mathbf{x})$ with the Dirichlet boundary conditions $\mathbf{x}(0)=\mathbf{0}=\mathbf{x}(1)$, where the vector field $\mathbf{f} \in C^{1}\left(\mathbb{R}^{n}, \mathbb{R}^{n}\right)$ is asymptotically linear and $\mathbf{f}(\mathbf{0})=\mathbf{0}$. We provide the existence and multiplicity results using the vector field rotation theory.

\section{Introduction}

The theory of nonlinear boundary value problems (BVPs in short) is intensively developed since the first works on calculus of variations where BVPs naturally appear in a classical problem of minimizing the integral functional considered on curves with fixed end points. The Euler equation for the problems of the calculus of variations can be written in the form

$$
x^{\prime \prime}=f\left(t, x, x^{\prime}\right)
$$

and the boundary conditions are

$$
\begin{aligned}
& x(a)=A, \\
& x(b)=B
\end{aligned}
$$

if the problem of fixed end points is considered. The methods for investigation of this problem are diverse. For the existence of a solution, a lot of papers use topological approaches. The main scheme is the following. Imagine $f$ in (1) is continuous and one is looking for classical $\left(x \in C^{2}([a, b])\right)$ solution of the problem. If $f$ is bounded, then problem (1) and (2) is solvable. This is true for scalar and vectorial cases. If $f$ is not bounded, then a priori estimates for a possible solution should be proved first in order to reduce given problem to that with bounded nonlinearity. The interested reader may consult books [1, Ch. 12] and [2-4] for details. We would like to mention also articles [5-8]. The diverse approaches to the subject were used in relatively recent contributions to the theory [9-16].

In all the above-mentioned references, the main question is about the existence of a solution. The problem of the uniqueness of a solution is the next important one, especially for purposes of numerical investigation. It is to be mentioned that both problems (existence and uniqueness) are closely related for linear problems. Indeed, the linear problem $x^{\prime \prime}+$ $k^{2} x=0, x(0)=A, x(1)=B$ has at most one solution for any $A, B \in \mathbb{R}$ if $k$ is not multiple of $\pi$. The condition $k \neq 0(\bmod \pi)$ is also sufficient for solvability of the problem for any $A, B$.

This is not the case for nonlinear problems. The solvability and multiplicity of solutions may be observed simultaneously. The problem $x^{\prime \prime}=-x^{3}, x(0)=0, x(1)=0$ is solvable and has a countable number of solutions. Another phenomenon was observed. Consider the problem $x^{\prime \prime}+f(x)=0$ together with Sturm-Liouville boundary conditions $a_{1} x(0)+a_{2} x^{\prime}(0)=$ $0, b_{1} x(1)-b_{2} x^{\prime}(1)=0$. It is convenient to look at this problem in a phase plane $\left(x, x^{\prime}\right)$. Suppose that $f(x) \approx k^{2} x$ at zero and $f(x) \approx l^{2} x$ at infinity, where $k$ and $l$ are essentially different constants. Then, the problem generally has multiple solutions due to the fact that trajectories of solutions of the equation have essentially different rotation speed near the origin and at infinity. This is evident geometrically and one of the first 
works employing this type of arguments is in the book [17, Ch. 15].

When passing to systems of the second-order differential equations, the analogous approach can be applied. The geometrical interpretation fails however. One should think of a substitute for the rotation (angular) speed. It appears that apparatus of vector fields is good enough. It is possible to construct special vector fields (based on the form of boundary conditions and on the behaviour of nonlinearities of a system) in the vicinity of the origin and "at infinity." This approach was applied to study BVPs for a system of the two secondorder nonlinear differential equations in the work [16]. The considered system was supposed to be asymptotically linear (of one kind) at zero and quasi-linear (linear plus bounded nonlinearity) of another kind at infinity. Special vector fields were considered and the appropriate rotation numbers were invented.

The current article considers the case of $n$ second-order differential equations. The approach is the same. However, there is need for employing the respective results concerning rotation of $n$-dimensional vector fields. The main object is a system of the second-order ordinary differential equations given together with the Dirichlet type boundary conditions. The main difference compared with paper [16] is that the computation of rotation numbers at zero and "at infinity" is more complicated and uses an advanced technique.

The structure of the work is the following. In Section 2, the general idea is discussed and useful references and needed definitions are given. In Section 3, the analysis of the vector field at zero (i.e., for solutions with small initial values) is carried out. The similar work is done in Section 4 for the infinity. Section 5 contains the main result. The example and the conclusions complete the article.

\section{The Vector Field $\phi$ Associated with the Dirichlet Boundary Value Problem}

Consider the system

$$
\mathbf{x}^{\prime \prime}=\mathbf{f}(\mathbf{x})
$$

given with the boundary conditions

$$
\mathbf{x}(0)=\mathbf{0}=\mathbf{x}(1)
$$

and the initial conditions

$$
\begin{gathered}
\mathbf{x}(0)=\mathbf{0}, \\
\mathbf{x}^{\prime}(0)=\boldsymbol{\beta},
\end{gathered}
$$

where $\mathbf{0}=(\underbrace{0,0, \ldots, 0}_{n})^{T} \in \mathbb{R}^{n}$.

We suppose that the following conditions are fulfilled.

(A1) $\mathbf{f} \in C^{1}\left(\mathbb{R}^{n}, \mathbb{R}^{n}\right)$.

(A2) $\mathbf{f}(\mathbf{0})=\mathbf{0}$, and hence system (3) has the trivial solution $\mathbf{x}=\mathbf{0}$.
(A3) The vector field $\mathbf{f}$ is asymptotically linear; that is, there exists $n \times n$ matrix $\mathbf{f}^{\prime}(\infty)$ with real entries such that

$$
\lim _{\|\mathbf{x}\| \rightarrow \infty} \frac{\left\|\mathbf{f}(\mathbf{x})-\mathbf{f}^{\prime}(\infty) \mathbf{x}\right\|}{\|\mathbf{x}\|}=0 .
$$

The norms are standard everywhere. The matrix $\mathbf{f}^{\prime}(\infty)$ is called the derivative of the vector field $\mathbf{f}$ at infinity [18].

It follows from the above conditions that

$$
\mathbf{f}(\mathbf{x})=\mathbf{f}^{\prime}(\infty) \mathbf{x}+\mathbf{h}(\mathbf{x}), \quad \forall \mathbf{x} \in \mathbb{R}^{n}
$$

where $\mathbf{h} \in C^{1}\left(\mathbb{R}^{n}, \mathbb{R}^{n}\right), \mathbf{h}(\mathbf{0})=\mathbf{0}$, and

$$
\lim _{\|\mathbf{x}\| \rightarrow \infty} \frac{\|\mathbf{h}(\mathbf{x})\|}{\|\mathbf{x}\|}=0 .
$$

It follows from (7) and (8) that the vector field $\mathbf{f}$ is asymptotically linear if and only if for any $\varepsilon>0$ there exists $M(\varepsilon)>0$ such that

$$
\|\mathbf{h}(\mathbf{x})\| \leq M(\varepsilon)+\varepsilon\|\mathbf{x}\|, \quad \forall \mathbf{x} \in \mathbb{R}^{n} .
$$

The asymptotically linear vector field $\mathbf{f}$ is linearly bounded. Indeed, fix $\varepsilon_{0}>0$ and consider the corresponding $M_{0}=$ $M\left(\varepsilon_{0}\right)>0$. Then, it follows from (7) and (9) that

$$
\begin{aligned}
\|\mathbf{f}(\mathbf{x})\| & \leq\left\|\mathbf{f}^{\prime}(\infty)\right\|\|\mathbf{x}\|+M_{0}+\varepsilon_{0}\|(\mathbf{x})\| \\
& =a_{1}+b_{1}\|\mathbf{x}\|, \quad \forall \mathbf{x} \in \mathbb{R}^{n},
\end{aligned}
$$

where $\left|\left\|\mathbf{f}^{\prime}(\infty)\right\|\right|=\max _{\|\boldsymbol{\beta}\|=1}\left\|\mathbf{f}^{\prime}(\infty) \boldsymbol{\beta}\right\| \geq 0, a_{1}=M_{0}>0$, and $b_{1}=\left|\left\|\mathbf{f}^{\prime}(\infty)\right\|\right|+\varepsilon_{0}>0$.

Rewrite system (3) in the equivalent form

$$
\mathbf{z}^{\prime}=\mathbf{F}(\mathbf{z})
$$

where $\mathbf{F}(\mathbf{z})=(\mathbf{y}, \mathbf{f}(\mathbf{x}))^{T}, \mathbf{z}=(\mathbf{x}, \mathbf{y})^{T} \in \mathbb{R}^{N}, \mathbf{y}=\mathbf{x}^{\prime}$, and $N=$ $2 n$.

Proposition 1. Suppose that conditions (A1), (A2), and (A3) are fulfilled. Then, the vector field $\mathbf{F}$ has the following properties.

(1) $\mathbf{F} \in C^{1}\left(\mathbb{R}^{N}, \mathbb{R}^{N}\right)$.

(2) $\mathbf{F}(\mathbf{o})=\mathbf{o} \in \mathbb{R}^{N}$, where $\mathbf{o}=(\mathbf{0}, \mathbf{0})^{T}$.

(3) The vector field $\mathbf{F}$ is asymptotically linear since there exists $N \times N$ matrix

$$
\mathbf{F}^{\prime}(\infty)=\left(\begin{array}{cc}
O_{n} & I_{n} \\
\mathbf{f}^{\prime}(\infty) & O_{n}
\end{array}\right),
$$

where $I_{n}$ and $O_{n}$ are $n \times n$ unity and zero matrices, respectively, such that

$$
\lim _{\|\mathbf{z}\|_{N} \rightarrow \infty} \frac{\left\|\mathbf{F}(\mathbf{z})-\mathbf{F}^{\prime}(\infty) \mathbf{z}\right\|_{N}}{\|\mathbf{z}\|_{N}}=0 .
$$

(4) The vector field $\mathbf{F}$ is linearly bounded. 
Proof. (1) and (2) follow from (A1) and (A2).

(3) For every $\mathbf{z}=(\mathbf{x}, \mathbf{y})^{T} \in \mathbb{R}^{N}$, one has that $\mathbf{F}(\mathbf{z})=$ $\mathbf{F}^{\prime}(\infty) \mathbf{z}+\mathbf{H}(\mathbf{z})$, where $\mathbf{H}(\mathbf{z})=(\mathbf{0}, \mathbf{h}(\mathbf{x}))^{T}$. Then, for any $\varepsilon>0$ there exists $M(\varepsilon)>0$ such that for every $\mathbf{z}=(\mathbf{x}, \mathbf{y})^{T} \in \mathbb{R}^{N}$

$$
\|\mathbf{H}(\mathbf{z})\|_{N}=\|\mathbf{h}(\mathbf{x})\| \leq M(\varepsilon)+\varepsilon\|\mathbf{x}\| \leq M(\varepsilon)+\varepsilon\|\mathbf{z}\|_{N} .
$$

(4) It follows that asymptotic linearity of the vector field $\mathbf{F}$ implies its linear boundedness.

Since the vector field $\mathbf{F} \in C^{1}\left(\mathbb{R}^{N}, \mathbb{R}^{N}\right)$ is linearly bounded, then $[19,20]$ its flow $\Phi^{t}(\gamma)=\mathbf{z}(t ; \gamma)$ is complete and $\boldsymbol{\Phi}^{t} \in C^{1}\left(\mathbb{R}^{N}, \mathbb{R}^{N}\right)$ for any $t \in \mathbb{R}$, where $\mathbf{z}(t ; \boldsymbol{\gamma})$ is the solution to the Cauchy problem

$$
\begin{aligned}
\mathbf{z}^{\prime} & =\mathbf{F}(\mathbf{z}), \\
\mathbf{z}(0) & =\boldsymbol{\gamma} .
\end{aligned}
$$

Let $\gamma=(\boldsymbol{\alpha}, \boldsymbol{\beta})^{T} \in \mathbb{R}^{N}$. We consider for our purposes the restriction of time one flow $\left.\Phi^{1}\right|_{\alpha=0}(\gamma)=\left(\mathbf{x}(1 ; \boldsymbol{\beta}), \mathbf{x}^{\prime}(1 ; \boldsymbol{\beta})\right)$, where $\mathbf{x}(1 ; \boldsymbol{\beta})$ is the solution to Cauchy problem (3) and (5). Denote the first component of $\left.\Phi^{1}\right|_{\boldsymbol{\alpha}=0}$ by $\phi$; that is,

$$
\phi(\boldsymbol{\beta})=\mathbf{x}(1 ; \boldsymbol{\beta}), \quad \forall \boldsymbol{\beta} \in \mathbb{R}^{n} .
$$

Then, $\phi \in C^{1}\left(\mathbb{R}^{n}, \mathbb{R}^{n}\right)$. The singular points of the vector field $\phi$ are $\boldsymbol{\beta} \in \mathbb{R}^{n}$ such that $\phi(\boldsymbol{\beta})=\mathbf{0}$ and they are in one-toone correspondence with the solutions to Dirichlet boundary value problem (3) and (4). It follows from condition (A2) that $\phi(\mathbf{0})=\mathbf{0}$ and hence the singular point $\boldsymbol{\beta}=\mathbf{0}$ of the vector field $\phi$ corresponds to the trivial solution to problem (3) and (4). Any singular point $\boldsymbol{\beta} \neq \mathbf{0}$ of the vector field $\phi$ generates a nontrivial solution to problem (3) and (4). In what follows, we investigate singular points of the vector field $\phi$ in terms of rotation numbers and provide the conditions which guarantee the existence of a solution (nontrivial) for the boundary value problem under consideration.

Consider a bounded open set $\Omega \subset \mathbb{R}^{n}$. Suppose that the vector field $\phi$ is nonsingular on the boundary $\partial \Omega$; that is,

$$
\phi(\boldsymbol{\beta}) \neq \mathbf{0}, \quad \forall \boldsymbol{\beta} \in \partial \Omega .
$$

Then [21, 22], there is an integer $\gamma(\phi, \Omega)$, which is associated with the vector field and called the rotation of the vector field $\phi$ on the boundary $\partial \Omega$.

A singular point $\boldsymbol{\beta}_{0} \in \mathbb{R}^{n}$ of the vector field $\phi$ is called isolated [21, 22], if there is neighbourhood $B_{r}\left(\boldsymbol{\beta}_{0}\right)=\{\| \boldsymbol{\beta}-$ $\left.\boldsymbol{\beta}_{0} \|<r, \boldsymbol{\beta} \in \mathbb{R}^{n}\right\}$ containing no other singular points. In this case, the rotation $\gamma\left(\boldsymbol{\phi}, B_{r}\left(\boldsymbol{\beta}_{0}\right)\right)$ is the same for any sufficiently small radius $r$. This common value ind $\left(\boldsymbol{\beta}_{0}, \boldsymbol{\phi}\right)$ is called the index of the isolated singular point $\boldsymbol{\beta}_{0} \in \mathbb{R}^{n}$.

If the vector field $\phi$ is nonsingular for all $\beta \in \mathbb{R}^{n}$ of sufficiently large norm, then by definition the point $\infty$ is an isolated singular point of $\phi$. In this case, the rotation $\gamma\left(\phi, B_{R}(\mathbf{0})\right)$ is the same for sufficiently large radius $R$. This common value ind $(\infty, \phi)$ is called the index of the isolated singular point $\infty[21,22]$.

\section{The Vector Field $\phi$ Near Zero}

Suppose that conditions (A1) and (A2) hold. Then, there exists the derivative $\mathbf{f}^{\prime}(\mathbf{0})$ (the Jacobian matrix) of the vector field $\mathbf{f}$ at zero $\mathbf{x}=\mathbf{0}$ and we can consider the linearized system at zero

$$
\mathbf{u}^{\prime \prime}=\mathbf{f}^{\prime}(\mathbf{0}) \mathbf{u},
$$

the Dirichlet boundary conditions

$$
\mathbf{u}(0)=\mathbf{0}=\mathbf{u}(1),
$$

and the initial conditions

$$
\begin{aligned}
\mathbf{u}(0) & =\mathbf{0}, \\
\mathbf{u}^{\prime}(0) & =\boldsymbol{\beta} .
\end{aligned}
$$

If $\mathbf{u}(t ; \boldsymbol{\beta})$ is a solution to Cauchy problem (18) and (20) and $P(t)$ is the solution to the $n \times n$ matrix Cauchy problem

$$
\begin{aligned}
P^{\prime \prime}(t) & =\mathbf{f}^{\prime}(\mathbf{0}) P(t), \\
P(0) & =O_{n}, \\
P^{\prime}(0) & =I_{n},
\end{aligned}
$$

then $\mathbf{u}(t ; \boldsymbol{\beta})=P(t) \boldsymbol{\beta}$ for every $t \in \mathbb{R}$ and $\boldsymbol{\beta} \in \mathbb{R}^{n}$. Let us define the linear vector field $\phi_{0}: \mathbb{R}^{n} \rightarrow \mathbb{R}^{n}$ :

$$
\phi_{0}(\boldsymbol{\beta})=\mathbf{u}(1 ; \boldsymbol{\beta})=P(1) \boldsymbol{\beta}, \quad \forall \boldsymbol{\beta} \in \mathbb{R}^{n} .
$$

Hence, $\boldsymbol{\phi}_{0}^{\prime}(\boldsymbol{\beta})=\boldsymbol{\phi}_{0}^{\prime}(\mathbf{0})=P(1)$ for every $\boldsymbol{\beta} \in \mathbb{R}^{n}$.

Let us consider the following condition.

(A4) The linearized system at zero (18) is nonresonant with respect to boundary conditions (19); that is, linear homogeneous problem (18) and (19) has only the trivial solution.

The spectrum $\sigma_{D}=\left\{-(j \pi)^{2}: j \in \mathbb{N}\right\}$ of the scalar Dirichlet boundary value problem

$$
\begin{aligned}
x^{\prime \prime} & =\lambda x, \\
x(0) & =0=x(1)
\end{aligned}
$$

consists of all $\lambda$ such that boundary value problem (23) has a nontrivial solution.

Proposition 2. The following statements are equivalent.

(1) Condition (A4) holds.

(2) $\operatorname{det} \phi_{0}^{\prime}(\mathbf{0})=\operatorname{det} P(1) \neq 0$.

(3) $\boldsymbol{\beta}=\mathbf{0}$ is the unique singular point of the vector field $\boldsymbol{\phi}_{0}$.

(4) No eigenvalue of matrix $\mathbf{f}^{\prime}(\mathbf{0})$ belongs to the spectrum $\sigma_{D}$ of scalar Dirichlet boundary value problem (23). 
Proof. The nonzero singular points of the vector field $\phi_{0}$ are in one-to-one correspondence with the nontrivial solutions to Dirichlet boundary value problem (18) and (19). Hence, the equivalence (1) $\Leftrightarrow(2) \Leftrightarrow$ (3) follows from (22).

Let us prove that $(2) \Leftrightarrow(4)$.

If $J$ is the real Jordan form [23] of matrix $\mathbf{f}^{\prime}(\mathbf{0})$, then there exists a real nonsingular matrix $M$ such that $J=M^{-1} \mathbf{f}^{\prime}(\mathbf{0}) M$. Cauchy problem (18) and (20) transforms to the Cauchy problem

$$
\begin{aligned}
\mathbf{v}^{\prime \prime} & =J \mathbf{v}, \\
\mathbf{v}(0) & =\mathbf{0}, \\
\mathbf{v}^{\prime}(0) & =\boldsymbol{\eta},
\end{aligned}
$$

where $\mathbf{v}=M^{-1} \mathbf{u}$ and $\boldsymbol{\eta}=M^{-1} \boldsymbol{\beta}$.

If $\mathbf{v}(t ; \boldsymbol{\eta})$ is the solution to Cauchy problem (24) and $Q(t)$ is the solution to the $n \times n$ matrix Cauchy problem

$$
\begin{gathered}
Q^{\prime \prime}(t)=J Q(t), \\
Q(0)=O_{n}, \\
Q^{\prime}(0)=I_{n},
\end{gathered}
$$

then $\mathbf{v}(t ; \boldsymbol{\eta})=Q(t) \boldsymbol{\eta}$ for every $t \in \mathbb{R}$ and $\boldsymbol{\eta} \in \mathbb{R}^{n}$. Let us consider the linear vector field $\psi_{0}: \mathbb{R}^{n} \rightarrow \mathbb{R}^{n}$ such that

$$
\boldsymbol{\psi}_{0}(\boldsymbol{\eta})=\mathbf{v}(1 ; \boldsymbol{\eta})=Q(1) \boldsymbol{\eta}, \quad \forall \boldsymbol{\eta} \in \mathbb{R}^{n}
$$

Hence, $\psi_{0}^{\prime}(\boldsymbol{\eta})=\psi_{0}^{\prime}(\mathbf{0})=Q(1)$ for every $\boldsymbol{\eta} \in \mathbb{R}^{n}$.

The Jacobian matrices $\phi_{0}^{\prime}(\boldsymbol{\beta})$ and $\psi_{0}^{\prime}(\boldsymbol{\eta})$ are similar. Indeed, since $\mathbf{v}(t ; \boldsymbol{\eta})=M^{-1} \mathbf{u}(t ; \boldsymbol{\beta})$ and $\boldsymbol{\eta}=M^{-1} \boldsymbol{\beta}$, one has that

$$
\begin{aligned}
\boldsymbol{\phi}_{0}^{\prime}(\boldsymbol{\beta}) & =M\left[\boldsymbol{\psi}_{0}(\boldsymbol{\eta})\right]_{\boldsymbol{\beta}}^{\prime}=M \boldsymbol{\psi}_{0}^{\prime}(\boldsymbol{\eta}) \boldsymbol{\eta}_{\boldsymbol{\beta}}^{\prime} \\
& =M \boldsymbol{\psi}_{0}^{\prime}(\boldsymbol{\eta}) M^{-1},
\end{aligned}
$$

and hence $P(1)=M Q(1) M^{-1}$ and $\operatorname{det} \phi_{0}^{\prime}(\mathbf{0})=\operatorname{det} P(1)=$ $\operatorname{det} Q(1)$. Next we shall analyze $\operatorname{det} Q(1)$.

The blocks of the real Jordan form $J$ of matrix $\mathbf{f}^{\prime}(\mathbf{0})$ are of two types [23]: a real eigenvalue $\lambda$ of matrix $\mathbf{f}^{\prime}(\mathbf{0})$ generates blocks

$$
J_{k}(\lambda)=\left(\begin{array}{cccccc}
\lambda & 1 & 0 & \cdots & 0 & 0 \\
0 & \lambda & 1 & \cdots & 0 & 0 \\
\vdots & \vdots & \vdots & \cdots & \vdots & \vdots \\
0 & 0 & 0 & \cdots & \lambda & 1 \\
0 & 0 & 0 & \cdots & 0 & \lambda
\end{array}\right)
$$

where $k$ is the size of the block, but a pair $\lambda=a+i b$ and $\bar{\lambda}=a-i b(b \neq 0)$ of complex conjugate eigenvalues of matrix $\mathbf{f}^{\prime}(\mathbf{0})$ is associated with blocks

$$
\begin{aligned}
J_{k}(\lambda)=C_{2 m}(\lambda) & \left(\begin{array}{cccccc}
C_{2}(\lambda) & I_{2} & O_{2} & \cdots & O_{2} & O_{2} \\
O_{2} & C_{2}(\lambda) & I_{2} & \cdots & O_{2} & O_{2} \\
\vdots & \vdots & \vdots & \cdots & \vdots & \vdots \\
O_{2} & O_{2} & O_{2} & \cdots & C_{2}(\lambda) & I_{2} \\
O_{2} & O_{2} & O_{2} & \cdots & O_{2} & C_{2}(\lambda)
\end{array}\right),
\end{aligned}
$$

where $k=2 m$ is the size of the block and

$$
\begin{aligned}
C_{2}(\lambda) & =\left(\begin{array}{ll}
a & -b \\
b & a
\end{array}\right), \\
I_{2} & =\left(\begin{array}{ll}
1 & 0 \\
0 & 1
\end{array}\right), \\
O_{2} & =\left(\begin{array}{ll}
0 & 0 \\
0 & 0
\end{array}\right) .
\end{aligned}
$$

Suppose $Q_{k}(t)=\left(q_{i j}(t)\right)$ solves the $k \times k$ matrix Cauchy problem

$$
\begin{aligned}
& Q_{k}^{\prime \prime}(t)=J_{k}(\lambda) Q_{k}(t), \\
& Q_{k}(0)=O_{k}, \\
& Q_{k}^{\prime}(0)=I_{k} .
\end{aligned}
$$

Let $\lambda$ be a real eigenvalue of matrix $\mathbf{f}^{\prime}(\mathbf{0})$ and $\lambda=r^{2} \operatorname{sgn} \lambda$, where $r=\sqrt{|\lambda|}$. Then $[15,24]$,

$$
\begin{aligned}
Q_{k}(t)= & I_{k} t+\frac{1}{3 !}\left[J_{k}(\lambda)\right] t^{3}+\frac{1}{5 !}\left[J_{k}(\lambda)\right]^{2} t^{5} \\
& +\frac{1}{7 !}\left[J_{k}(\lambda)\right]^{3} t^{7}+\cdots \\
& +\frac{1}{(2 j+1) !}\left[J_{k}(\lambda)\right]^{j} t^{2 j+1}+\cdots \\
= & \sum_{j=0}^{\infty} \frac{1}{(2 j+1) !}\left[J_{k}(\lambda)\right]^{j} t^{2 j+1} .
\end{aligned}
$$




$$
\left[J_{k}(\lambda)\right]^{j}=\left(\begin{array}{cccccc}
r^{2 j}(\operatorname{sgn} \lambda)^{j} & * & * & \cdots & * & * \\
0 & r^{2 j}(\operatorname{sgn} \lambda)^{j} & * & \cdots & * & * \\
\vdots & \vdots & \vdots & \cdots & \vdots & \vdots \\
0 & 0 & 0 & \cdots & r^{2 j}(\operatorname{sgn} \lambda)^{j} & * \\
0 & 0 & 0 & \cdots & 0 & r^{2 j}(\operatorname{sgn} \lambda)^{j}
\end{array}\right)
$$

are upper triangular matrices, then matrix $Q_{k}(t)$ is upper triangular also with the diagonal elements

$$
q(t)=\sum_{j=0}^{\infty} \frac{1}{(2 j+1) !} r^{2 j}(\operatorname{sgn} \lambda)^{j} t^{2 j+1} .
$$

It follows from (31) that function $q(t)=q_{k k}(t)$ solves the Cauchy problem

$$
\begin{aligned}
q^{\prime \prime}(t) & =r^{2} \operatorname{sgn} \lambda q(t), \\
q(0) & =0, \\
q^{\prime}(0) & =1, \\
\operatorname{det} Q_{k}(1) & =[q(1)]^{k} .
\end{aligned}
$$

(a) If $\lambda=0$, then the solution to the Cauchy problem

$$
\begin{gathered}
q^{\prime \prime}(t)=0, \\
q(0)=0, \\
q^{\prime}(0)=1
\end{gathered}
$$

is $q(t)=t$. Hence,

$$
\operatorname{det} Q_{k}(1)=[q(1)]^{k}=1^{k}=1>0 .
$$

(b) If $\lambda=r^{2}>0(r>0)$, then the solution to the Cauchy problem

$$
\begin{aligned}
q^{\prime \prime}(t) & =r^{2} q(t), \\
q(0) & =0, \\
q^{\prime}(0) & =1
\end{aligned}
$$

is $q(t)=\sinh (r t) / r$. Hence,

$$
\operatorname{det} Q_{k}(1)=[q(1)]^{k}=\left[\frac{\sinh r}{r}\right]^{k}=\frac{\sinh ^{k} r}{r^{k}}>0 .
$$

(c) If $\lambda=-r^{2}<0(r>0)$, then the solution to the Cauchy problem

$$
\begin{aligned}
q^{\prime \prime}(t) & =-r^{2} q(t), \\
q(0) & =0, \\
q^{\prime}(0) & =1
\end{aligned}
$$

is $q(t)=\sin (r t) / r$. Hence,

$$
\begin{aligned}
\operatorname{det} Q_{k}(1) & =[q(1)]^{k}=\left[\frac{\sin r}{r}\right]^{k}=\frac{\sin ^{k} r}{r^{k}} \neq 0 \Longleftrightarrow \lambda \\
& \notin \sigma_{D} .
\end{aligned}
$$

(d) Suppose $\lambda=a+i b$ and $\bar{\lambda}=a-i b(b \neq 0)$ are complex conjugate eigenvalues of matrix $\mathbf{f}^{\prime}(\mathbf{0})$ and $J_{k}(\lambda)=C_{2 m}(\lambda)$, $k=2 m$. Then $[15,24]$,

$$
\begin{aligned}
Q_{2 m}(t)= & I_{2 m} t+\frac{1}{3 !}\left[C_{2 m}(\lambda)\right] t^{3}+\frac{1}{5 !}\left[C_{2 m}(\lambda)\right]^{2} t^{5} \\
& +\frac{1}{7 !}\left[C_{2 m}(\lambda)\right]^{3} t^{7}+\cdots \\
& +\frac{1}{(2 j+1) !}\left[C_{2 m}(\lambda)\right]^{j} t^{2 j+1}+\cdots \\
= & \sum_{j=0}^{\infty} \frac{1}{(2 j+1) !}\left[C_{2 m}(\lambda)\right]^{j} t^{2 j+1} .
\end{aligned}
$$

The matrices

$$
\begin{aligned}
& {\left[J_{k}(\lambda)\right]^{j}=\left[C_{2 m}(\lambda)\right]^{j}} \\
& =\left(\begin{array}{cccccc}
{\left[C_{2}(\lambda)\right]^{j}} & * & * & \cdots & * & * \\
O_{2} & {\left[C_{2}(\lambda)\right]^{j}} & * & \cdots & * & * \\
\vdots & \vdots & \vdots & \cdots & \vdots & \vdots \\
O_{2} & O_{2} & O_{2} & \cdots & {\left[C_{2}(\lambda)\right]^{j}} & * \\
O_{2} & O_{2} & O_{2} & \cdots & O_{2} & {\left[C_{2}(\lambda)\right]^{j}}
\end{array}\right)
\end{aligned}
$$

are $k \times k$ upper triangular block matrices of $2 \times 2$ blocks,

$$
\left[C_{2}(\lambda)\right]^{j}=\left(\begin{array}{cc}
a & -b \\
b & a
\end{array}\right)^{j}=\left(\begin{array}{cc}
\rho^{j} \cos j \varphi & -\rho^{j} \sin j \varphi \\
\rho^{j} \sin j \varphi & \rho^{j} \cos j \varphi
\end{array}\right),
$$


where $a=\rho \cos \varphi$ and $b=\rho \sin \varphi$. Then, $Q_{2 m}(t)$ is $k \times k$ upper triangular block matrix of $2 \times 2$ blocks also with diagonal blocks

$$
\begin{aligned}
D_{2}(t)= & \left(\begin{array}{cc}
u(t) & -v(t) \\
v(t) & u(t)
\end{array}\right) \\
= & I_{2} t+\frac{1}{3 !}\left[C_{2}(\lambda)\right] t^{3}+\frac{1}{5 !}\left[C_{2}(\lambda)\right]^{2} t^{5} \\
& +\frac{1}{7 !}\left[C_{2}(\lambda)\right]^{3} t^{7}+\cdots \\
& +\frac{1}{(2 j+1) !}\left[C_{2}(\lambda)\right]^{j} t^{2 j+1}+\cdots \\
= & \sum_{j=0}^{\infty} \frac{1}{(2 j+1) !}\left[C_{2}(\lambda)\right]^{j} t^{2 j+1},
\end{aligned}
$$

where

$$
\begin{aligned}
& u(t)=\sum_{j=0}^{\infty} \frac{\rho^{j} \cos j \varphi}{(2 j+1) !} t^{2 j+1}, \\
& v(t)=\sum_{j=0}^{\infty} \frac{\rho^{j} \sin j \varphi}{(2 j+1) !} t^{2 j+1} .
\end{aligned}
$$

It follows from (31) that the matrix

$$
D_{2}(t)=\left(\begin{array}{cc}
u(t) & -v(t) \\
v(t) & u(t)
\end{array}\right)=\left(\begin{array}{cc}
q_{k-1, k-1}(t) & q_{k-1, k}(t) \\
q_{k, k-1}(t) & q_{k, k}(t)
\end{array}\right)
$$

solves the matrix Cauchy problem

$$
\begin{aligned}
& D_{2}^{\prime \prime}(t)=C_{2}(\lambda) D_{2}(t), \\
& D_{2}(0)=O_{2}, \\
& D_{2}^{\prime}(0)=I_{2}
\end{aligned}
$$

or

$$
\begin{aligned}
\left(\begin{array}{ll}
u^{\prime \prime}(t) & -v^{\prime \prime}(t) \\
v^{\prime \prime}(t) & u^{\prime \prime}(t)
\end{array}\right) & =\left(\begin{array}{ll}
a & -b \\
b & a
\end{array}\right)\left(\begin{array}{cc}
u(t) & -v(t) \\
v(t) & u(t)
\end{array}\right), \\
\left(\begin{array}{cc}
u(0) & -v(0) \\
v(0) & u(0)
\end{array}\right) & =\left(\begin{array}{ll}
0 & 0 \\
0 & 0
\end{array}\right), \\
\left(\begin{array}{ll}
u^{\prime}(0) & -v^{\prime}(0) \\
v^{\prime}(0) & u^{\prime}(0)
\end{array}\right) & =\left(\begin{array}{ll}
1 & 0 \\
0 & 1
\end{array}\right) .
\end{aligned}
$$

Suppose that $\lambda=a+i b=\mu^{2}=(\alpha+i \beta)^{2}$, where $a=\alpha^{2}-\beta^{2}$ and $b=2 \alpha \beta \neq 0(\alpha \neq 0, \beta \neq 0)$. Then, functions $u(t)$ and $v(t)$ solve the Cauchy problem

$$
\begin{aligned}
u^{\prime \prime}(t) & =\left(\alpha^{2}-\beta^{2}\right) u(t)-(2 \alpha \beta) v(t), \\
v^{\prime \prime}(t) & =(2 \alpha \beta) u(t)+\left(\alpha^{2}-\beta^{2}\right) v(t), \\
u(0) & =0, \\
u^{\prime}(0) & =1, \\
v(0) & =0, \\
v^{\prime}(0) & =0,
\end{aligned}
$$

and hence

$$
\begin{aligned}
u(t) & =\frac{1}{\alpha^{2}+\beta^{2}}[\alpha \sinh (\alpha t) \cos (\beta t) \\
& +\beta \cosh (\alpha t) \sin (\beta t)],
\end{aligned}
$$

$$
\begin{aligned}
v(t) & =\frac{1}{\alpha^{2}+\beta^{2}}[\alpha \cosh (\alpha t) \sin (\beta t) \\
& -\beta \sinh (\alpha t) \cos (\beta t)] .
\end{aligned}
$$

Therefore,

$$
\begin{aligned}
\operatorname{det} D_{2}(1) & =\left|\begin{array}{cc}
u(1) & -v(1) \\
v(1) & u(1)
\end{array}\right|=u^{2}(1)+v^{2}(1) \\
& =\frac{\sinh ^{2} \alpha \cos ^{2} \beta+\cosh ^{2} \alpha \sin ^{2} \beta}{\alpha^{2}+\beta^{2}}>0, \\
\operatorname{det} Q_{2 m}(1) & =\left[\operatorname{det} D_{2}(1)\right]^{m} \\
& =\frac{\left(\sinh ^{2} \alpha \cos ^{2} \beta+\cosh ^{2} \alpha \sin ^{2} \beta\right)^{m}}{\left(\alpha^{2}+\beta^{2}\right)^{m}}>0 .
\end{aligned}
$$

The determinant of $Q(1)$ is equal to the product of the determinants of the blocks $Q_{k}(1)$ corresponding to the eigenvalues $\lambda$ of matrix $\mathbf{f}^{\prime}(\mathbf{0})$. It follows from the abovementioned considerations that $\operatorname{det} \phi_{0}^{\prime}(\mathbf{0})=\operatorname{det} P(1)=$ $\operatorname{det} Q(1) \neq 0$ if and only if the eigenvalues of matrix $\mathbf{f}^{\prime}(\mathbf{0})$ do not belong to the spectrum $\sigma_{D}=\left\{-(j \pi)^{2}: j \in \mathbb{N}\right\}$ of scalar Dirichlet boundary value problem (23). Hence, (2) $\Leftrightarrow$ (4).

Proposition 3. Suppose that condition (A4) holds. If matrix $\mathbf{f}^{\prime}(\mathbf{0})$ does not have negative eigenvalues with odd algebraic multiplicities, then ind $\left(\mathbf{0}, \boldsymbol{\phi}_{0}\right)=1$. If matrix $\mathbf{f}^{\prime}(\mathbf{0})$ has $k(1 \leq$ $k \leq n)$ different negative eigenvalues $\lambda_{j}(1 \leq j \leq k)$ with odd algebraic multiplicities, then

$$
\begin{aligned}
\operatorname{ind}\left(\mathbf{0}, \boldsymbol{\phi}_{0}\right) & =\operatorname{sgn} \operatorname{det} \boldsymbol{\phi}_{0}^{\prime}(\mathbf{0})=\operatorname{sgn} \operatorname{det} P(1) \\
& =\operatorname{sgn}\left(\prod_{j=1}^{k} \sin \sqrt{\left|\lambda_{j}\right|}\right) .
\end{aligned}
$$


Proof. Suppose that condition (A4) holds. It follows from Proposition 2 that $\operatorname{det} \phi_{0}^{\prime}(\mathbf{0})=\operatorname{det} P(1) \neq 0$ and $\boldsymbol{\beta}=\mathbf{0}$ is the unique singular point of the vector field $\phi_{0}$. Hence $[21,22]$,

$$
\text { ind }\left(\mathbf{0}, \boldsymbol{\phi}_{0}\right)=\operatorname{sgn} \operatorname{det} \boldsymbol{\phi}_{0}^{\prime}(\mathbf{0})=\operatorname{sgn} \operatorname{det} Q(1) .
$$

The sign of $\operatorname{det} Q(1)$ is equal to the product of the signs of $\operatorname{det} Q_{k}(1)$ for the blocks $Q_{k}(1)$ corresponding to the eigenvalues $\lambda$ of matrix $\mathbf{f}^{\prime}(\mathbf{0})$. It follows from the proof of Proposition 2 that $\operatorname{sgn} \operatorname{det} Q_{k}(1)=1$ for the blocks $Q_{k}(1)$ corresponding to nonnegative and complex eigenvalues $\lambda$ of matrix $\mathbf{f}^{\prime}(\mathbf{0})$. Let $\lambda=-r^{2}<0(r=\sqrt{|\lambda|})$ be a negative eigenvalue of matrix $\mathbf{f}^{\prime}(\mathbf{0})$ with algebraic multiplicity $\mu$ and geometric multiplicity $\gamma, 1 \leq \gamma \leq \mu \leq n$. Then, matrix $Q(1)$ has $\gamma$ blocks $Q_{k_{1}}(1), \ldots, Q_{k_{\gamma}}(1)$ corresponding to the eigenvalue $\lambda$ and

$$
\begin{aligned}
\operatorname{det} & Q_{k_{1}}(1) \cdot \ldots \cdot \operatorname{det} Q_{k_{\gamma}}(1)=\frac{\sin ^{k_{1}} r}{r^{k_{1}}} \cdot \ldots \cdot \frac{\sin ^{k_{\gamma}} r}{r^{k_{\gamma}}} \\
= & \frac{\sin ^{k_{1}+\cdots+k_{\gamma}} r}{r^{k_{1}+\cdots+k_{\gamma}}}=\frac{\sin ^{\mu} r}{r^{\mu}} \neq 0 .
\end{aligned}
$$

Therefore,

$$
\begin{aligned}
& \text { sgn } \operatorname{det} Q_{k_{1}}(1) \cdot \ldots \cdot \text { sgn } \operatorname{det} Q_{k_{\gamma}}(1) \\
& \quad= \begin{cases}+1, & \text { if } \mu \text { is even, } \\
\operatorname{sgn} \sin \sqrt{|\lambda|}, & \text { if } \mu \text { is odd. }\end{cases}
\end{aligned}
$$

If matrix $\mathbf{f}^{\prime}(\mathbf{0})$ does not have negative eigenvalues with odd algebraic multiplicities, then ind $\left(\mathbf{0}, \phi_{0}\right)=1$. If matrix $\mathbf{f}^{\prime}(\mathbf{0})$ has $k(1 \leq k \leq n)$ different negative eigenvalues $\lambda_{j}(1 \leq j \leq$ $k$ ) with odd algebraic multiplicities, then formula (53) is valid.

Theorem 4. Suppose that conditions (A1), (A2), and (A4) hold. Then, $\boldsymbol{\beta}=\mathbf{0}$ is an isolated singular point of the vector field $\boldsymbol{\phi}$ and ind $(\mathbf{0}, \boldsymbol{\phi})=$ ind $\left(\mathbf{0}, \boldsymbol{\phi}_{0}\right)$.

Proof. We already mentioned that the flow $\Phi^{t}(\gamma)=\mathbf{z}(t ; \gamma)$ of the vector field $\mathbf{F}$ is of class $C^{1}$ for every $t \in \mathbb{R}$, where $\mathbf{z}(t ; \gamma)$ is the solution to Cauchy problem (15). Then, there exist continuous partial derivatives $\left(\partial z_{i} / \partial \gamma_{j}\right)(t ; \gamma)(i, j=$ $1,2, \ldots, N)$ for every $t \in \mathbb{R}$ and $\gamma \in \mathbb{R}^{N}$. Matrix $Z(t ; \gamma)=$ $\left(\left(\partial z_{i} / \partial \gamma_{j}\right)(t ; \gamma)\right)$ solves $[4,19]$ the $N \times N$ matrix Cauchy problem

$$
\begin{aligned}
& Z^{\prime}(t ; \boldsymbol{\gamma})=\mathbf{F}^{\prime}(\mathbf{z}(t ; \boldsymbol{\gamma})) Z(t ; \boldsymbol{\gamma}), \\
& Z(0 ; \boldsymbol{\gamma})=I_{N},
\end{aligned}
$$

where $\mathbf{F}^{\prime}(\mathbf{z}(t ; \boldsymbol{\gamma}))$ is the Jacobian matrix of the vector field $\mathbf{F}$ along the solution $\mathbf{z}(t ; \boldsymbol{\gamma})$. One has for $\gamma=(\mathbf{0}, \boldsymbol{\beta})$, taking into account that $\mathbf{z}=\left(\mathbf{x}, \mathbf{x}^{\prime}\right)$, that matrix $X(t ; \boldsymbol{\beta})=$ $\left(\left(\partial x_{i} / \partial \beta_{j}\right)(t ; \boldsymbol{\beta})\right)$ solves the $n \times n$ matrix Cauchy problem

$$
\begin{aligned}
X^{\prime \prime}(t ; \boldsymbol{\beta}) & =\mathbf{f}^{\prime}(\mathbf{x}(t ; \boldsymbol{\beta})) X(t ; \boldsymbol{\beta}), \\
X(0 ; \boldsymbol{\beta}) & =O_{n}, \\
X^{\prime}(0 ; \boldsymbol{\beta}) & =I_{n},
\end{aligned}
$$

where $\mathbf{f}^{\prime}(\mathbf{x}(t ; \boldsymbol{\beta}))$ is the Jacobian matrix of the vector field $\mathbf{f}$ along the solution $\mathbf{x}(t ; \boldsymbol{\beta})$ to Cauchy problem (3) and (5). If $\boldsymbol{\beta}=\mathbf{0}$, then it follows from condition (A2) that $\mathbf{x}(t ; \mathbf{0})=\mathbf{0}$ and the matrix $X(t ; \mathbf{0})=\left(\left(\partial x_{i} / \partial \beta_{j}\right)(t ; \mathbf{0})\right)$ solves the $n \times n$ matrix Cauchy problem

$$
\begin{aligned}
X^{\prime \prime}(t ; \mathbf{0}) & =\mathbf{f}^{\prime}(\mathbf{0}) X(t ; \mathbf{0}), \\
X(0 ; \mathbf{0}) & =O_{n}, \\
X^{\prime}(0 ; \mathbf{0}) & =I_{n} .
\end{aligned}
$$

Uniqueness of solutions to $n \times n$ matrix Cauchy problems (21) and (59) implies that $X(t ; \mathbf{0})=P(t)$ for every $t \in \mathbb{R}$. Hence, $X(1 ; \mathbf{0})=P(1)$. Notice that $X(1 ; \mathbf{0})=\left(\left(\partial x_{i} / \partial \beta_{j}\right)(1 ; \mathbf{0})\right)=$ $\phi^{\prime}(\mathbf{0})$. Therefore, $\phi^{\prime}(\mathbf{0})=P(1)$. Since $P(1)=\phi_{0}^{\prime}(\mathbf{0})$, one has that $\phi^{\prime}(\mathbf{0})=\phi_{0}^{\prime}(\mathbf{0})$. It follows from Proposition 2 that $\operatorname{det} \phi^{\prime}(\mathbf{0})=\operatorname{det} \phi_{0}^{\prime}(\mathbf{0}) \neq 0$. Hence, $[21,22] \boldsymbol{\beta}=\mathbf{0}$ is an isolated singular point of the vector field $\phi$ and

$$
\text { ind } \begin{aligned}
(\mathbf{0}, \boldsymbol{\phi}) & =\operatorname{sgn} \operatorname{det} \boldsymbol{\phi}^{\prime}(\mathbf{0})=\operatorname{sgn} \operatorname{det} \boldsymbol{\phi}_{0}^{\prime}(\mathbf{0}) \\
& =\text { ind }\left(\mathbf{0}, \boldsymbol{\phi}_{0}\right) .
\end{aligned}
$$

\section{The Vector Field $\phi$ at Infinity}

Suppose that conditions (A1) and (A3) hold. Then, there exists the derivative $\mathbf{f}^{\prime}(\infty)$ of the vector field $\mathbf{f}$ at infinity and we can consider the linearized system at infinity

$$
\mathbf{w}^{\prime \prime}=\mathbf{f}^{\prime}(\infty) \mathbf{w}
$$

the Dirichlet boundary conditions

$$
\mathbf{w}(0)=\mathbf{0}=\mathbf{w}(1)
$$

and the initial conditions

$$
\begin{aligned}
\mathbf{w}(0) & =\mathbf{0}, \\
\mathbf{w}^{\prime}(0) & =\boldsymbol{\beta} .
\end{aligned}
$$

If $\mathbf{w}(t ; \boldsymbol{\beta})$ is the solution to Cauchy problem (61) and (63) and $S(t)$ is the solution to $n \times n$ matrix Cauchy problem

$$
\begin{aligned}
& S^{\prime \prime}(t)=\mathbf{f}^{\prime}(\infty) S(t), \\
& S(0)=O_{n}, \\
& S^{\prime}(0)=I_{n},
\end{aligned}
$$

then $\mathbf{w}(t ; \boldsymbol{\beta})=S(t) \boldsymbol{\beta}$ for every $t \in \mathbb{R}$ and $\boldsymbol{\beta} \in \mathbb{R}^{n}$. Let us define the linear vector field $\phi_{\infty}: \mathbb{R}^{n} \rightarrow \mathbb{R}^{n}$,

$$
\phi_{\infty}(\boldsymbol{\beta})=\mathbf{w}(1 ; \boldsymbol{\beta})=S(1) \boldsymbol{\beta}, \quad \forall \boldsymbol{\beta} \in \mathbb{R}^{n} .
$$

Hence, $\phi_{\infty}^{\prime}(\boldsymbol{\beta})=\phi_{\infty}^{\prime}(\mathbf{0})=S(1)$ for every $\boldsymbol{\beta} \in \mathbb{R}^{n}$. 
Let us consider the following condition.

(A5) The linearized system at infinity (61) is nonresonant with respect to boundary conditions (62); that is, linear homogeneous problem (61) and (62) has only the trivial solution.

Proposition 5. The following statements are equivalent.

(1) Condition (A5) holds.

(2) $\operatorname{det} \phi_{\infty}^{\prime}(\mathbf{0})=\operatorname{det} S(1) \neq 0$.

(3) $\boldsymbol{\beta}=\mathbf{0}$ is the unique singular point of the vector field $\phi_{\infty}$.

(4) No eigenvalue of the matrix $\mathbf{f}^{\prime}(\infty)$ belongs to the spectrum $\sigma_{D}$ of scalar Dirichlet boundary value problem (23).

Proposition 6. Suppose that condition (A5) holds. If the matrix $\mathbf{f}^{\prime}(\infty)$ does not have negative eigenvalues with odd algebraic multiplicities, then ind $\left(\mathbf{0}, \phi_{\infty}\right)=1$. If the matrix $\mathbf{f}^{\prime}(\infty)$ has $s(1 \leq s \leq n)$ different negative eigenvalues $\mu_{i}(1 \leq$ $i \leq s)$ with odd algebraic multiplicities, then

$$
\begin{aligned}
\operatorname{ind}\left(\mathbf{0}, \boldsymbol{\phi}_{\infty}\right) & =\operatorname{sgn} \operatorname{det} \phi_{\infty}^{\prime}(\mathbf{0})=\operatorname{sgn} \operatorname{det} S(1) \\
& =\operatorname{sgn}\left(\prod_{i=1}^{s} \sin \sqrt{\left|\mu_{i}\right|}\right) .
\end{aligned}
$$

The proofs of Propositions 5 and 6 are analogous to the proofs of Propositions 2 and 3, respectively.

Theorem 7. Suppose that conditions (A1), (A3), and (A5) hold. Then, the point $\infty$ is an isolated singular point of the vector field $\phi$ and ind $(\infty, \phi)=$ ind $\left(\mathbf{0}, \phi_{\infty}\right)$.

Proof. First of all, we shall prove that the vector field $\phi$ is asymptotically linear with the derivative at infinity $\phi^{\prime}(\infty)=$ $\boldsymbol{\phi}_{\infty}^{\prime}(\mathbf{0})=S(1)$. We proceed in the following steps.

Step 1 (auxiliary linear nonhomogeneous initial value problem). Let us consider the function $\mathbf{p}(t ; \boldsymbol{\beta})=(1 /\|\boldsymbol{\beta}\|) \mathbf{x}(t ; \boldsymbol{\beta})-$ $\mathbf{w}(t ; \boldsymbol{\beta} /\|\boldsymbol{\beta}\|)$ for every $t \in \mathbb{R}$ and $\boldsymbol{\beta} \in \mathbb{R}^{n} \backslash\{\mathbf{0}\}$, where $\mathbf{x}(t ; \boldsymbol{\beta})$ is the solution to Cauchy problem (3) and (5) and $\mathbf{w}(t ; \boldsymbol{\beta})$ is the solution to Cauchy problem (61) and (63). The function $\mathbf{p}(t ; \boldsymbol{\beta})$ solves the Cauchy problem

$$
\begin{aligned}
\mathbf{p}^{\prime \prime}(t ; \boldsymbol{\beta}) & =\mathbf{f}^{\prime}(\infty) \mathbf{p}(t ; \boldsymbol{\beta})+\mathbf{g}(t ; \boldsymbol{\beta}) \\
\mathbf{p}(0 ; \boldsymbol{\beta}) & =\mathbf{0} \\
\mathbf{p}^{\prime}(0 ; \boldsymbol{\beta}) & =\mathbf{0}
\end{aligned}
$$

where $\mathbf{g}(t ; \boldsymbol{\beta})=(1 /\|\boldsymbol{\beta}\|) \mathbf{h}(\mathbf{x}(t ; \boldsymbol{\beta}))$ for every $t \in \mathbb{R}$ and $\boldsymbol{\beta} \epsilon$ $\mathbb{R}^{n} \backslash\{\mathbf{0}\}$. One can find $[24,25]$ that

$$
\begin{aligned}
& \mathbf{p}(t ; \boldsymbol{\beta})=\sum_{k=0}^{\infty}\left[\mathbf{f}^{\prime}(\infty)\right]^{k} \boldsymbol{\varphi}_{2 k+1}(t ; \boldsymbol{\beta}) \\
& \boldsymbol{\varphi}_{m}(t ; \boldsymbol{\beta})=\int_{0}^{t} \frac{(t-\tau)^{m}}{m !} \mathbf{g}(\tau ; \boldsymbol{\beta}) d \tau, \\
& (m=0,1,2, \ldots), \\
& \varphi_{1}^{\prime}(t ; \beta)=\varphi_{0}(t ; \beta), \\
& \varphi_{3}^{\prime}(t ; \beta)=\varphi_{2}(t ; \beta), \\
& \varphi_{5}^{\prime}(t ; \boldsymbol{\beta})=\varphi_{4}(t ; \boldsymbol{\beta}), \\
& \boldsymbol{\varphi}_{1}^{\prime \prime}(t ; \boldsymbol{\beta})=\mathbf{g}(t ; \boldsymbol{\beta}), \\
& \varphi_{3}^{\prime \prime}(t ; \beta)=\varphi_{1}(t ; \beta) \\
& \boldsymbol{\varphi}_{5}^{\prime \prime}(t ; \boldsymbol{\beta})=\varphi_{3}(t ; \boldsymbol{\beta}),
\end{aligned}
$$

Step 2 (estimates for $\left.\left\|\boldsymbol{\varphi}_{m}(1 ; \boldsymbol{\beta})\right\|(m=0,1,2, \ldots)\right)$. Suppose $\boldsymbol{\beta} \neq \mathbf{0}$ and consider

$$
\begin{array}{r}
\boldsymbol{\varphi}_{m}(1 ; \boldsymbol{\beta})=\int_{0}^{1} \frac{(t-\tau)^{m}}{m !} \mathbf{g}(\tau ; \boldsymbol{\beta}) d \tau \\
\quad(m=0,1,2, \ldots) .
\end{array}
$$

Taking into account (9) for any $\varepsilon>0$, one concludes that there exists $M(\varepsilon)>0$ such that

$$
\|\mathbf{h}(\mathbf{x}(\tau ; \boldsymbol{\beta}))\| \leq M(\varepsilon)+\varepsilon\|\mathbf{x}(\tau ; \boldsymbol{\beta})\|, \quad \forall \tau \in[0,1] .
$$

Since $0 \leq 1-\tau \leq 1$, one has

$$
\begin{aligned}
\left\|\frac{(1-\tau)^{m}}{m !} \mathbf{g}(\tau ; \boldsymbol{\beta})\right\| & =\frac{|1-\tau|^{m}}{m !} \frac{\|\mathbf{h}(\mathbf{x}(\tau ; \boldsymbol{\beta}))\|}{\|\boldsymbol{\beta}\|} \\
& \leq \frac{1}{m !\|\boldsymbol{\beta}\|}\|\mathbf{h}(\mathbf{x}(\tau ; \boldsymbol{\beta}))\| \\
& \leq \frac{1}{m !\|\boldsymbol{\beta}\|}(M(\varepsilon)+\varepsilon\|\mathbf{x}(\tau ; \boldsymbol{\beta})\|), \\
& \forall \tau \in[0,1] .
\end{aligned}
$$

In accordance with Proposition 1, the vector field $\mathbf{F}$ is asymptotically linear, and hence there exist $A, B \geq 0$ such that 
$\|\mathbf{F}(\mathbf{z})\| \leq A+B\|\mathbf{z}\|$ for every $\mathbf{z}=(\mathbf{x}, \mathbf{y})^{T} \in \mathbb{R}^{N}, \mathbf{y}=\mathbf{x}^{\prime}$. Consider the integral equation $\mathbf{z}(\tau ; \boldsymbol{\gamma})=\boldsymbol{\gamma}+\int_{0}^{\tau} \mathbf{F}(\mathbf{z}(s ; \boldsymbol{\gamma})) d s$ equivalent to the Cauchy problem $\mathbf{z}^{\prime}(\tau ; \boldsymbol{\gamma})=\mathbf{F}(\mathbf{z}(\tau ; \boldsymbol{\gamma})), \mathbf{z}(0 ; \boldsymbol{\gamma})=\gamma=$ $(\mathbf{0}, \boldsymbol{\beta})^{T}$. Then,

$$
\|\mathbf{z}(\tau ; \gamma)\| \leq\|\gamma\|+A \tau+B \int_{0}^{\tau}\|\mathbf{z}(s ; \gamma)\| d s .
$$

Using Grönwall's inequality [20], one has that

$$
\|\mathbf{z}(\tau ; \gamma)\| \leq(\|\gamma\|+A \tau) e^{B \tau} .
$$

Therefore,

$$
\|\mathbf{z}(\tau ; \boldsymbol{\gamma})\| \leq A e^{B}+e^{B}\|\boldsymbol{\gamma}\|, \quad \forall \tau \in[0,1] .
$$

Since $\|\mathbf{x}(\tau ; \boldsymbol{\beta})\| \leq\|\mathbf{z}(\tau ; \boldsymbol{\gamma})\|$ and $\|\boldsymbol{\gamma}\|=\|\boldsymbol{\beta}\|$, we obtain

$$
\|\mathbf{x}(\tau ; \boldsymbol{\beta})\| \leq A e^{B}+e^{B}\|\boldsymbol{\beta}\|, \quad \forall \tau \in[0,1] .
$$

It follows from (72) and (76) that

$$
\left\|\frac{(1-\tau)^{m}}{m !} \mathbf{g}(\tau ; \boldsymbol{\beta})\right\| \leq \frac{M(\varepsilon)+\varepsilon A e^{B}}{m !\|\boldsymbol{\beta}\|}+\frac{\varepsilon e^{B}}{m !},
$$

$\forall \tau \in[0,1]$.

Therefore,

$$
\begin{aligned}
\left\|\boldsymbol{\varphi}_{m}(1 ; \boldsymbol{\beta})\right\| & =\left\|\int_{0}^{1} \frac{(1-\tau)^{m}}{m !} \mathbf{g}(\tau ; \boldsymbol{\beta}) d \tau\right\| \\
& \leq \int_{0}^{1}\left\|\frac{(1-\tau)^{m}}{m !} \mathbf{g}(\tau ; \boldsymbol{\beta})\right\| d \tau \\
& \leq \frac{M(\varepsilon)+\varepsilon A e^{B}}{m !\|\boldsymbol{\beta}\|}+\frac{\varepsilon e^{B}}{m !}, \\
\left\|\boldsymbol{\varphi}_{m}(1 ; \boldsymbol{\beta})\right\| & \leq \frac{M(\varepsilon)+\varepsilon A e^{B}}{m !\|\boldsymbol{\beta}\|}+\frac{\varepsilon e^{B}}{m !} .
\end{aligned}
$$

Step 3. Let us prove that $\lim _{\|\boldsymbol{\beta}\| \rightarrow \infty}\|\mathbf{p}(1 ; \boldsymbol{\beta})\|=0$.

(1) Suppose that $\mathbf{f}^{\prime}(\infty)=O_{n}$. It follows from (68) and (78) that

$$
\|\mathbf{p}(1 ; \boldsymbol{\beta})\|=\left\|\boldsymbol{\varphi}_{1}(1 ; \boldsymbol{\beta})\right\| \leq \frac{M(\varepsilon)+\varepsilon A e^{B}}{\|\boldsymbol{\beta}\|}+\varepsilon e^{B} .
$$

Hence,

$$
\lim _{\|\boldsymbol{\beta}\| \rightarrow \infty}\|\mathbf{p}(1 ; \boldsymbol{\beta})\| \leq \varepsilon e^{B} .
$$

Since $\varepsilon>0$ can be arbitrary, $\lim _{\|\boldsymbol{\beta}\| \rightarrow \infty}\|\mathbf{p}(1 ; \boldsymbol{\beta})\|=0$.

(2) Suppose that $\mathscr{B}=\mathbf{f}^{\prime}(\infty) \neq O_{n}$. Then,

$$
|\|\mathscr{B}\||=\max _{\|\beta\|=1}\|\mathscr{B} \beta\|>0 .
$$

Let us prove that the series $\sum_{k=0}^{\infty} \mathscr{B}^{k} \boldsymbol{\varphi}_{2 k+1}(1 ; \boldsymbol{\beta})$ is absolutely convergent; that is, the number series $\sum_{k=0}^{\infty}\left\|\mathscr{B}^{k} \boldsymbol{\varphi}_{2 k+1}(1 ; \boldsymbol{\beta})\right\|$ is convergent. It follows from (78) that

$$
\begin{gathered}
\left\|\mathscr{B}^{k} \boldsymbol{\varphi}_{2 k+1}(1 ; \boldsymbol{\beta})\right\| \leq\left\|\mathscr{B}^{k}\right\|\left\|\boldsymbol{\varphi}_{2 k+1}(1 ; \boldsymbol{\beta})\right\| \\
\leq \frac{\mid\|\mathscr{B}\|^{k}}{(2 k+1) !}\left(\frac{M(\varepsilon)+\varepsilon A e^{B}}{\|\boldsymbol{\beta}\|}+\varepsilon e^{B}\right) .
\end{gathered}
$$

The series $\sum_{k=0}^{\infty}\left(\|\| \mathscr{B} \|^{k} /(2 k+1) !\right)\left(\left(M(\varepsilon)+\varepsilon A e^{B}\right) /\|\beta\|+\varepsilon e^{B}\right)$ converges and the sum is

$$
\begin{aligned}
\sum_{k=0}^{\infty} \frac{|\|\mathscr{B}\||^{k}}{(2 k+1) !}\left(\frac{M(\varepsilon)+\varepsilon A e^{B}}{\|\beta\|}+\varepsilon e^{B}\right) \\
\quad=\frac{\sinh (\sqrt{|\|\mathscr{B}\||})}{\sqrt{|\|\mathscr{B}\||}}\left(\frac{M(\varepsilon)+\varepsilon A e^{B}}{\|\boldsymbol{\beta}\|}+\varepsilon e^{B}\right) .
\end{aligned}
$$

One can conclude from (82) by using the comparison test that the number series $\sum_{k=0}^{\infty}\left\|\mathscr{B}^{k} \boldsymbol{\varphi}_{2 k+1}(1 ; \boldsymbol{\beta})\right\|$ is convergent also and the sum is

$$
\begin{aligned}
& \sum_{k=0}^{\infty}\left\|\mathscr{B}^{k} \boldsymbol{\varphi}_{2 k+1}(1 ; \boldsymbol{\beta})\right\| \\
& \quad \leq \frac{\sinh (\sqrt{|\|\mathscr{B}\||})}{\sqrt{\mid\|\mathscr{B}\|}}\left(\frac{M(\varepsilon)+\varepsilon A e^{B}}{\|\boldsymbol{\beta}\|}+\varepsilon e^{B}\right) .
\end{aligned}
$$

Hence, the series $\sum_{k=0}^{\infty} \mathscr{B}^{k} \boldsymbol{\varphi}_{2 k+1}(1 ; \boldsymbol{\beta})$ is absolutely convergent and

$$
\begin{aligned}
\|\mathbf{p}(1 ; \boldsymbol{\beta})\| & =\left\|\sum_{k=0}^{\infty} \mathscr{B}^{k} \boldsymbol{\varphi}_{2 k+1}(1 ; \boldsymbol{\beta})\right\| \\
& \leq \sum_{k=0}^{\infty}\left\|\mathscr{B}^{k} \boldsymbol{\varphi}_{2 k+1}(1 ; \boldsymbol{\beta})\right\| \\
& \leq \frac{\sinh (\sqrt{|\|\mathscr{B}\||})}{\sqrt{\mid\|\mathscr{B}\|}}\left(\frac{M(\varepsilon)+\varepsilon A e^{B}}{\|\boldsymbol{\beta}\|}+\varepsilon e^{B}\right) .
\end{aligned}
$$

Therefore, $\lim _{\|\boldsymbol{\beta}\| \rightarrow \infty}\|\mathbf{p}(1 ; \boldsymbol{\beta})\| \leq \varepsilon e^{B}(\sinh (\sqrt{|\|\mathscr{R}\||}) / \sqrt{|\|\mathscr{R}\||})$. Since $\varepsilon>0$ can be chosen arbitrary, one has that $\lim _{\|\boldsymbol{\beta}\| \rightarrow \infty}\|\mathbf{p}(1 ; \boldsymbol{\beta})\|=0$.

Step 4 (asymptotic linearity of the vector field $\boldsymbol{\phi}$ ). If $\boldsymbol{\beta} \neq \mathbf{0}$, then

$$
\begin{aligned}
\left\|\phi(\boldsymbol{\beta})-\phi_{\infty}(\boldsymbol{\beta})\right\| & =\|\mathbf{x}(1 ; \boldsymbol{\beta})-\mathbf{w}(1 ; \boldsymbol{\beta})\| \\
& =\|\mathbf{x}(1 ; \boldsymbol{\beta})-S(1) \boldsymbol{\beta}\| \\
& =\|\mathbf{x}(1 ; \boldsymbol{\beta})-\| \boldsymbol{\beta} \| S(1) \frac{\boldsymbol{\beta}}{\|\boldsymbol{\beta}\| \|} \\
& =\|\mathbf{x}(1 ; \boldsymbol{\beta})-\| \boldsymbol{\beta}\left\|\mathbf{w}\left(1 ; \frac{\boldsymbol{\beta}}{\|\boldsymbol{\beta}\|}\right)\right\| \\
& =\|\boldsymbol{\beta}\|\left\|\frac{1}{\|\boldsymbol{\beta}\|} \mathbf{x}(1 ; \boldsymbol{\beta})-\mathbf{w}\left(1 ; \frac{\boldsymbol{\beta}}{\|\boldsymbol{\beta}\|}\right)\right\| \\
& =\|\boldsymbol{\beta}\|\|\mathbf{p}(1 ; \boldsymbol{\beta})\| .
\end{aligned}
$$


Hence,

$$
\begin{aligned}
\frac{\left\|\phi(\beta)-\phi_{\infty}(\boldsymbol{\beta})\right\|}{\|\boldsymbol{\beta}\|} & =\frac{\left\|\phi(\boldsymbol{\beta})-\phi_{\infty}^{\prime}(\mathbf{0}) \boldsymbol{\beta}\right\|}{\|\boldsymbol{\beta}\|} \\
& =\|\mathbf{p}(1 ; \boldsymbol{\beta})\| .
\end{aligned}
$$

Since $\lim _{\|\boldsymbol{\beta}\| \rightarrow \infty}\|\mathbf{p}(1 ; \boldsymbol{\beta})\|=0$,

$$
\lim _{\|\boldsymbol{\beta}\| \rightarrow \infty} \frac{\left\|\phi(\boldsymbol{\beta})-\boldsymbol{\phi}_{\infty}^{\prime}(\mathbf{0}) \boldsymbol{\beta}\right\|}{\|\boldsymbol{\beta}\|}=0 ;
$$

that is, the vector field $\phi$ is asymptotically linear with the derivative at infinity $\phi^{\prime}(\infty)=\phi_{\infty}^{\prime}(\mathbf{0})$. It follows from Proposition 5 that $\operatorname{det} \phi^{\prime}(\infty)=\operatorname{det} \phi_{\infty}^{\prime}(\mathbf{0}) \neq 0$. Hence $[21,22]$, the point $\infty$ is an isolated singular point of the vector field $\phi$ and

$$
\begin{aligned}
\operatorname{ind}(\infty, \phi) & =\operatorname{sgn} \operatorname{det} \phi^{\prime}(\infty)=\operatorname{sgn} \operatorname{det} \phi_{\infty}^{\prime}(\mathbf{0}) \\
& =\operatorname{ind}\left(\mathbf{0}, \phi_{\infty}\right) .
\end{aligned}
$$

\section{The Main Theorem}

Let us recall that the singular points of the vector field $\phi$ are in one-to-one correspondence with solutions to Dirichlet boundary value problem (3) and (4). A solution $\mathbf{x}(t ; \boldsymbol{\beta})$ of problem (3) and (4) is called nondegenerate, if the singular point $\beta$ of the vector field $\phi$ is nondegenerate; that is, $\operatorname{det} \phi^{\prime}(\beta) \neq 0$.

Theorem 8. Suppose that conditions (A1) to (A5) hold. Then, the points $\boldsymbol{\beta}=\mathbf{0}$ and $\infty$ are isolated singular points of the vector field $\phi$.

(a) If ind $(\mathbf{0}, \phi) \neq$ ind $(\infty, \phi)$, then boundary value problem (3) and (4) has a nontrivial solution.

(b) If ind $(\mathbf{0}, \phi) \neq$ ind $(\infty, \phi)$ and boundary value problem (3) and (4) has a nontrivial nondegenerate solution, then there exists yet another nontrivial solution to problem (3) and (4).

Proof. (a) It follows from Theorems 4 and 7 that the points $\boldsymbol{\beta}=\mathbf{0}$ and $\infty$ are isolated singular points of the vector field $\phi$. Hence, one can find positive $r, R$ such that $r<R$ and the sets

$$
\begin{aligned}
\bar{B}_{r}(\mathbf{0}) \backslash\{\mathbf{0}\} & =\left\{0<\|\boldsymbol{\beta}\| \leq r, \boldsymbol{\beta} \in \mathbb{R}^{n}\right\}, \\
\bar{B}_{R}(\infty) & =\left\{\|\boldsymbol{\beta}\| \geq R, \boldsymbol{\beta} \in \mathbb{R}^{n}\right\}
\end{aligned}
$$

contain no singular points of the vector field $\phi$. The vector field $\boldsymbol{\phi}$ is nonsingular on the spheres $S_{r}(\mathbf{0})=\partial B_{r}(\mathbf{0})$ and $S_{R}(\mathbf{0})=\partial B_{R}(\mathbf{0})$ and the rotations on these spheres are different:

$$
\begin{aligned}
\gamma\left(\boldsymbol{\phi}, B_{r}(\mathbf{0})\right) & =\operatorname{ind}(\mathbf{0}, \boldsymbol{\phi}) \neq \operatorname{ind}(\boldsymbol{\infty}, \boldsymbol{\phi}) \\
& =\gamma\left(\boldsymbol{\phi}, B_{R}(\mathbf{0})\right) .
\end{aligned}
$$

Using [22, Theorem 2], one can conclude that the $n$ dimensional annulus

$$
\operatorname{Ann}(r, R)=\left\{r<\|\boldsymbol{\beta}\|<R, \boldsymbol{\beta} \in \mathbb{R}^{n}\right\}
$$

contains a singular point $\boldsymbol{\beta}_{0} \neq \mathbf{0}$ of the vector field $\boldsymbol{\phi}$, which generates a nontrivial solution to Dirichlet boundary value problem (3) and (4).

(b) Let ind $(\mathbf{0}, \boldsymbol{\phi}) \neq$ ind $(\infty, \boldsymbol{\phi})$ and suppose $\mathbf{x}\left(t ; \boldsymbol{\beta}_{0}\right)$ is a nontrivial nondegenerate solution to boundary value problem (3) and (4), or equivalently $\boldsymbol{\beta}_{0} \in \mathbb{R}^{n}$ is a nonzero nondegenerate singular point of the vector field $\phi$. Then $[21,22]$, ind $\left(\boldsymbol{\beta}_{0}, \boldsymbol{\phi}\right)=\operatorname{sgn} \operatorname{det} \phi^{\prime}\left(\boldsymbol{\beta}_{0}\right) \in\{-1,1\}$. Suppose the contrary that $\mathbf{x}\left(t ; \boldsymbol{\beta}_{0}\right)$ is the unique nontrivial solution to boundary value problem (3) and (4) or equivalently $\boldsymbol{\beta}_{0}$ is the unique singular point of the vector field $\boldsymbol{\phi}$ in the set $\mathbb{R}^{n} \backslash\{\mathbf{0}\}$. Hence $[21,22]$,

$$
\operatorname{ind}(\infty, \phi)=\operatorname{ind}(\mathbf{0}, \boldsymbol{\phi})+\operatorname{ind}\left(\boldsymbol{\beta}_{0}, \boldsymbol{\phi}\right) \text {. }
$$

If ind $(\infty, \phi)=1$ and ind $(\mathbf{0}, \boldsymbol{\phi})=-1$, then ind $\left(\boldsymbol{\beta}_{0}, \boldsymbol{\phi}\right)=$ ind $(\infty, \phi)-$ ind $(\mathbf{0}, \phi)=1-(-1)=2$. If ind $(\infty, \phi)=-1$ and ind $(\mathbf{0}, \boldsymbol{\phi})=1$, then ind $\left(\boldsymbol{\beta}_{0}, \boldsymbol{\phi}\right)=$ ind $(\infty, \boldsymbol{\phi})-\operatorname{ind}(\mathbf{0}, \boldsymbol{\phi})=$ $-1-1=-2$. The contradiction proves that there exists a singular point $\boldsymbol{\beta}_{1} \in \mathbb{R}^{n} \backslash\{\mathbf{0}\}$ of the vector field $\boldsymbol{\phi}$ such that $\boldsymbol{\beta}_{1} \neq \boldsymbol{\beta}_{0}$ or equivalently that there exists a solution $\mathbf{x}\left(t ; \boldsymbol{\beta}_{1}\right)$ to boundary value problem (3) and (4), which is different from $\mathbf{x}\left(t ; \boldsymbol{\beta}_{0}\right)$.

Remark 9. The practical implementation of Theorem 8 is based on Propositions 3 and 6 and Theorems 4 and 7. Firstly the eigenvalues of the matrices $\mathbf{f}^{\prime}(\mathbf{0})$ and $\mathbf{f}^{\prime}(\boldsymbol{\infty})$ must be calculated. If the eigenvalues do not belong to spectrum $\sigma_{D}=\left\{-(j \pi)^{2}: j \in \mathbb{N}\right\}$ of scalar Dirichlet boundary value problem (23), then the indices ind $(\mathbf{0}, \boldsymbol{\phi})$ and ind $(\infty, \boldsymbol{\phi})$ must be calculated accordingly with Propositions 3 and 6 . If these indices are different, then Theorem 8 is applicable and the existence of a nontrivial solution to boundary value problem (3) and (4) can be concluded.

Remark 10. Suppose that conditions (A1) to (A5) hold and ind $(0, \phi) \neq$ ind $(\infty, \phi)$. If boundary value problem (3) and (4) has an odd number of nontrivial nondegenerate solutions $\mathbf{x}\left(t ; \boldsymbol{\beta}_{i}\right)(i=0,1, \ldots, 2 k)$, where $\boldsymbol{\beta}_{i} \neq \mathbf{0}$ and $\operatorname{det} \phi^{\prime}\left(\boldsymbol{\beta}_{i}\right) \neq$ $0(i=0,1, \ldots, 2 k)$, then there exists yet another nontrivial solution to problem (3) and (4). Suppose the contrary that the set $\mathbb{R}^{n} \backslash\{\mathbf{0}\}$ contains only an odd number of singular points $\boldsymbol{\beta}_{i}(i=0,1, \ldots, 2 k)$ of the vector field $\phi$ and these points are nondegenerate. Then $[21,22]$, ind $(\infty, \phi)-$ ind $(\mathbf{0}, \boldsymbol{\phi})=$ $\sum_{i=0}^{2 k}$ ind $\left(\boldsymbol{\beta}_{i}, \boldsymbol{\phi}\right)$, where the left hand side is equal to \pm 2 , but the right hand side is odd.

\section{Example}

Consider the system

$$
\begin{aligned}
& x_{1}^{\prime \prime}=-k^{2} \arctan \left(x_{1}+x_{2}\right), \\
& x_{2}^{\prime \prime}=-m^{2} \arctan \left(x_{1}-x_{2}\right),
\end{aligned}
$$


where $k$ and $m$ are nonzero integers, together with the boundary conditions

$$
x_{1}(0)=x_{2}(0)=0=x_{1}(1)=x_{2}(1) .
$$

Consider the vector field $\mathbf{f}: \mathbb{R}^{2} \rightarrow \mathbb{R}^{2}$ :

$$
\begin{array}{r}
\mathbf{f}(\mathbf{x}) \\
=\left(-k^{2} \arctan \left(x_{1}+x_{2}\right),-m^{2} \arctan \left(x_{1}-x_{2}\right)\right)^{T}, \\
\forall \mathbf{x}=\left(x_{1}, x_{2}\right)^{T} \in \mathbb{R}^{2} .
\end{array}
$$

Obviously conditions (A1) and (A2) are fulfilled. Due to the boundedness of arctan function, the vector field $\mathbf{f}$ is asymptotically linear and $\mathbf{f}^{\prime}(\infty)=\mathrm{O}_{2}$, and hence condition (A3) is fulfilled also.

Matrix $\mathbf{f}^{\prime}(\infty)$ has the only eigenvalue $\mu=0 \notin \sigma_{D}$, and hence matrix $\mathbf{f}^{\prime}(\infty)$ does not have negative eigenvalues with odd algebraic multiplicities. It follows from Proposition 6 and Theorem 7 that ind $(\infty, \phi)=1$. equation

The matrix $\mathbf{f}^{\prime}(\mathbf{0})=\left(\begin{array}{cc}-k^{2} & -k^{2} \\ -m^{2} & m^{2}\end{array}\right)$ has the characteristic

$$
p(\lambda)=\lambda^{2}-\left(m^{2}-k^{2}\right) \lambda-2 k^{2} m^{2}=0
$$

and the eigenvalues are

$$
\lambda_{1,2}=\frac{m^{2}-k^{2}}{2} \pm \frac{\sqrt{\left(k^{2}+m^{2}\right)^{2}+4 k^{2} m^{2}}}{2} .
$$

Since $\sqrt{\left(k^{2}+m^{2}\right)^{2}+4 k^{2} m^{2}}>k^{2}+m^{2}>m^{2}-k^{2}$, one has that $\lambda_{1}>0$ and $\lambda_{2}<0$. Obviously $\lambda_{1} \notin \sigma_{D}=\left\{-(j \pi)^{2}\right.$ : $j \in \mathbb{N}\}$. Note that $\lambda_{2} \notin \sigma_{D}$ also since $\lambda_{2}$ is an algebraic number $\left(\lambda_{2}\right.$ is the root of the characteristic polynomial $p(\lambda)$ with rational coefficients), but the spectrum $\sigma_{D}$ consists of the transcendental numbers (the product $-(j \pi)^{2}$ of the algebraic number $-j^{2}$ with the transcendental number $\pi^{2}$ is transcendental number). Theorem 8 is applicable, taking into account Proposition 3 and Theorem 4 , if ind $(\mathbf{0}, \boldsymbol{\phi})=$ $\operatorname{sgn} \sin \sqrt{\left|\lambda_{2}\right|}=-1$. Hence, Theorem 8 guarantees the existence of a nontrivial solution to boundary value problem (94) and (95) for all nonzero integers $k$ and $m$ such that

$$
\sin \sqrt{\frac{\sqrt{\left(k^{2}+m^{2}\right)^{2}+4 k^{2} m^{2}}}{2}-\frac{m^{2}-k^{2}}{2}}<0 .
$$

The pairs $(k, m)(1 \leq k, m \leq 10)$ with integer coordinates which satisfy condition (99) are depicted in Figure 1.

\section{Conclusions}

For an asymptotically linear system of $n$ the second-order ordinary differential equations that are assumed to have the trivial solution to the conditions for existence of nontrivial solutions of the Dirichlet boundary value problem are given. The technique and concepts of the theory of rotation of $n$ dimensional vector fields are used. The existence conditions

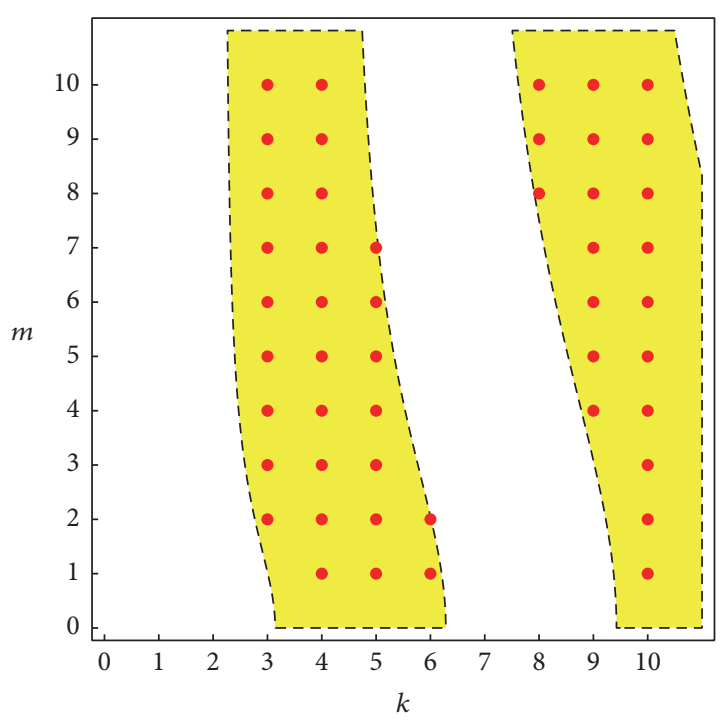

FIGURE 1: The points $(k, m)(1 \leq k, m \leq 10)$ with integer coordinates such that $k$ and $m$ satisfy condition (99).

are formulated in terms of eigenvalues of coefficient matrices of linearized systems at zero (at the trivial solution) and at infinity. The proposed approach is applicable to other twopoint boundary conditions such as the Neumann problem and mixed problem.

\section{Competing Interests}

The authors declare that there is no conflict of interests regarding the publication of this paper.

\section{References}

[1] P. Hartman, Ordinary Differential Equations, John Wiley \& Sons, New York, NY, USA, 1964.

[2] S. R. Bernfeld and V. Lakshmikantham, An Introduction to Nonlinear Boundary Value Problems, Academic Press, New York, NY, USA, 1974.

[3] N. I. Vasilyev and Y. A. Klokov, Foundations of the Theory of Boundary Value Problems for Ordinary Differential Equations, Apgads Zinatne, Rīga, Latvia, 1978 (Russian).

[4] W. G. Kelley and A. C. Peterson, The Theory of Differential Equations: Classical and Qualitative, Springer, 2010.

[5] R. Conti, "Equazioni differenziali ordinarie quasilineari con condizioni lineari," Annali di Matematica Pura ed Applicata, vol. 57, no. 1, pp. 49-61, 1962.

[6] P. Hartman, "On boundary value problems for systems of ordinary, nonlinear, second order differential equations," Transactions of the American Mathematical Society, vol. 96, no. 3, pp. 493-509, 1960.

[7] J. Mawhin, "Boundary value problems for nonlinear secondorder vector differential equations," Journal of Differential Equations, vol. 16, no. 2, pp. 257-269, 1974.

[8] W. T. Reid, "Comparison theorems for non-linear vector differential equations," Journal of Differential Equations, vol. 5, no. 2, pp. 324-337, 1969. 
[9] A. Abbondandolo, Morse Theory for Hamiltonian Systems, Chapman \& Hall/CRC, 2001.

[10] A. Capietto, F. Dalbono, and A. Portaluri, "A multiplicity result for a class of strongly indefinite asymptotically linear second order systems," Nonlinear Analysis: Theory, Methods \& Applications, vol. 72, no. 6, pp. 2874-2890, 2010.

[11] A. Capietto, W. Dambrosio, and D. Papini, "Detecting multiplicity for systems of second-order equations: an alternative approach," Advances in Differential Equations, vol. 10, no. 5, pp. 553-578, 2005.

[12] Y. Dong, "Index theory, nontrivial solutions, and asymptotically linear second-order Hamiltonian systems," Journal of Differential Equations, vol. 214, no. 2, pp. 233-255, 2005.

[13] A. Margheri and C. Rebelo, "Multiplicity of solutions of asymptotically linear Dirichlet problems associated to second order equations in $\mathrm{R}^{2 n+1}$," Topological Methods in Nonlinear Analysis, vol. 46, no. 2, pp. 1107-1118, 2015.

[14] D. O'Regan and R. Precup, Theorems of Leray-Schauder Type and Applications, CRC Press, New York, NY, USA, 2002.

[15] R. B. Taher and M. Rachidi, "Linear matrix differential equations of higher-order and applications," Electronic Journal of Differential Equations, vol. 2008, no. 95, pp. 1-12, 2008.

[16] I. Yermachenko and F. Sadyrbaev, "On a problem for a system of two second-order differential equations via the theory of vector fields," Nonlinear Analysis: Modelling and Control, vol. 20, no. 2, pp. 217-226, 2015.

[17] M. A. Krasnosel'skiǔ, A. I. Perov, A. I. Povolotskiy, and P. P. Zabreiko, Plane Vector Fields, Academic Press, New York, NY, USA, 1966.

[18] M. A. Krasnosel'skii, The Operator of Translation Along the Trajectories of Differential Equations, American Mathematical Society, Providence, RI, USA, 1968.

[19] V. I. Arnold, Ordinary Differential Equations, Springer, Berlin, Germany, 1992.

[20] E. Zehnder, Lectures on Dynamical Systems: Hamiltonian Vector Fields and Symplectic Capacities, European Mathematical Society, Helsinki, Finland, 2010.

[21] M. A. Krasnosel'skii and P. P. Zabreı̌ko, Geometrical Methods of Nonlinear Analysis, Springer, Berlin, Germany, 1984.

[22] P. P. Zabrejko, "Rotation of vector fields: definition, basic properties, and calculation," in Topological Nonlinear Analysis II: Degree, Singularity and Variations, M. Matzeu and A. Vignoli, Eds., vol. 27 of Progress in Nonlinear Differential Equations and Their Applications, pp. 445-601, Birkhäuser, Boston, Mass, USA, 1997.

[23] R. A. Horn and C. R. Johnson, Matrix Analysis, Cambridge University Press, London, UK, 1990.

[24] F. R. Gantmacher, The Theory of Matrices, AMS Chelsea Publishing, Providence, RI, USA, 1998.

[25] V. A. Zorich, Mathematical Analysis I, Springer, Berlin, Germany, 2004. 


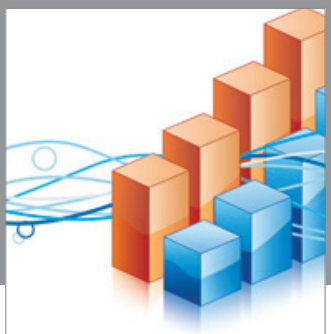

Advances in

Operations Research

vatem alat4

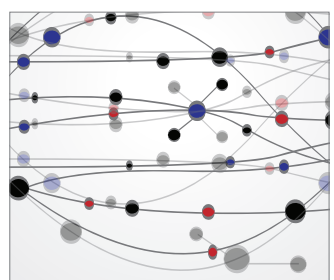

\section{The Scientific} World Journal
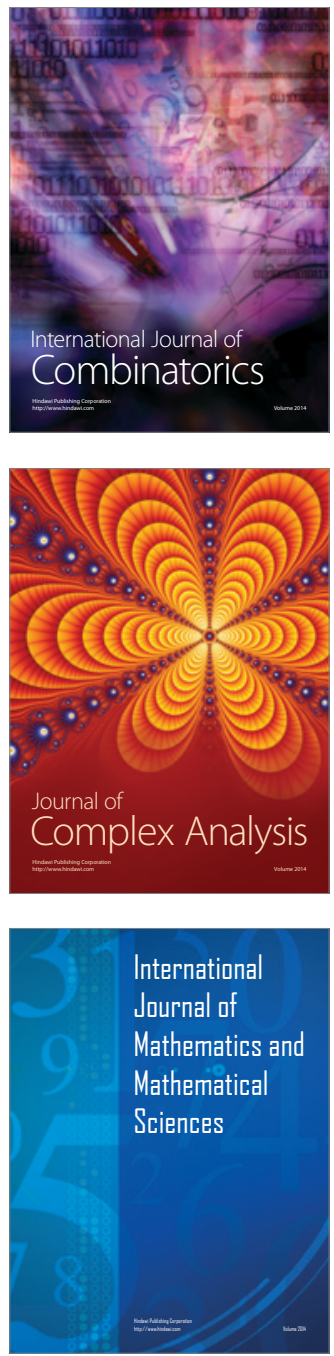
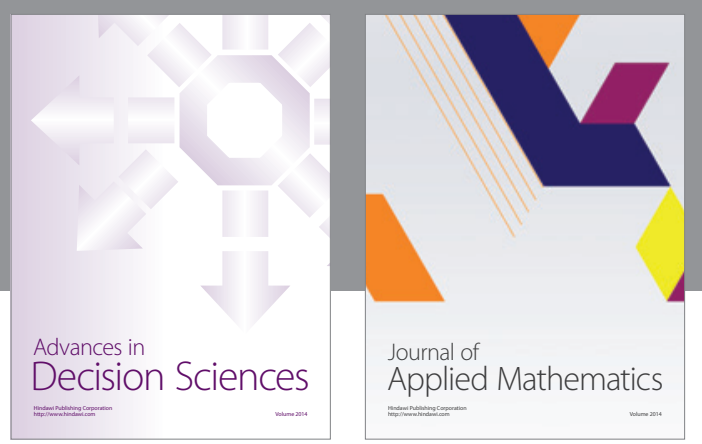

Algebra

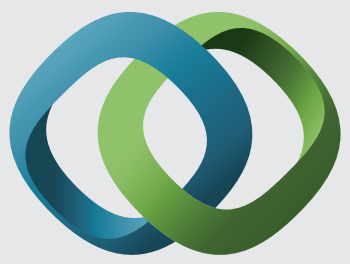

\section{Hindawi}

Submit your manuscripts at

http://www.hindawi.com
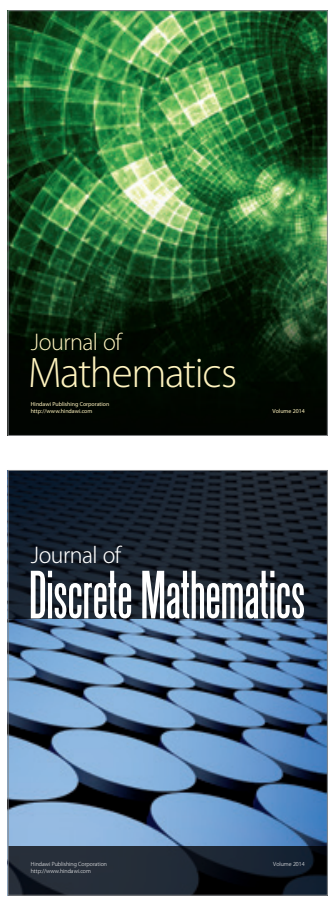

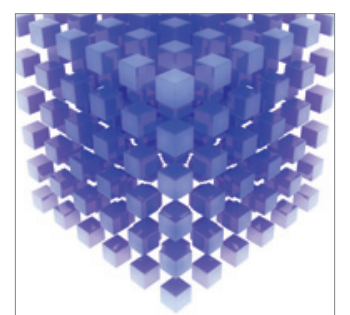

Mathematical Problems in Engineering
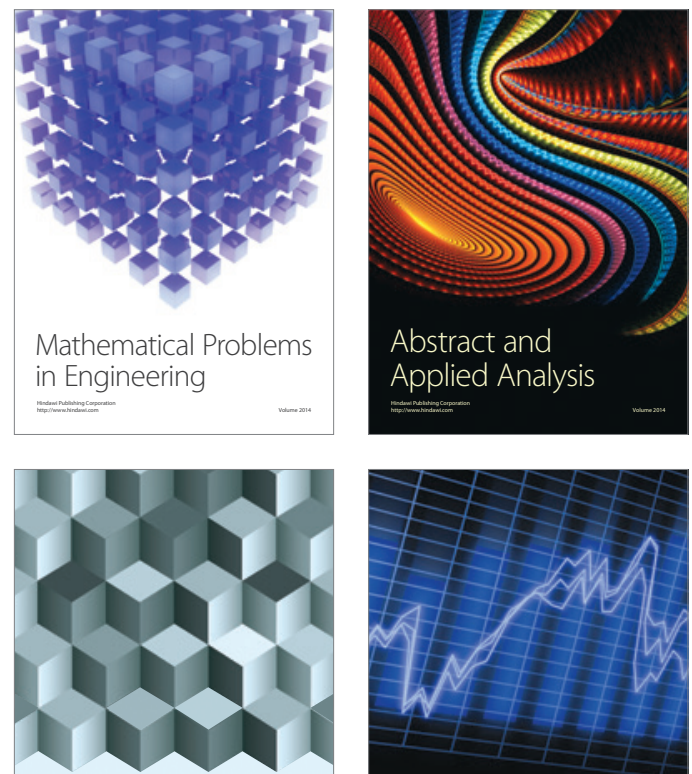

Journal of

Function Spaces

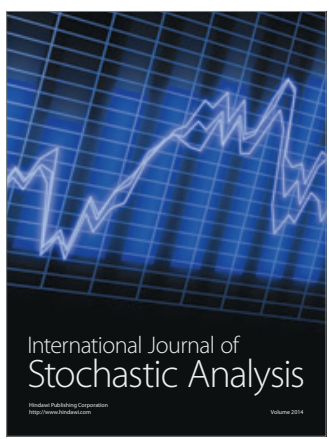

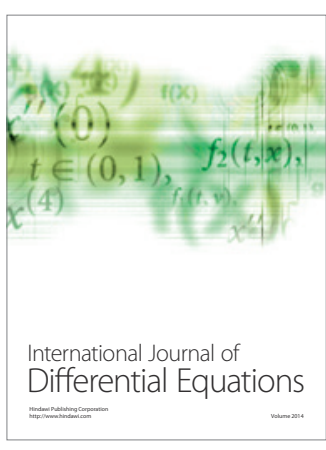
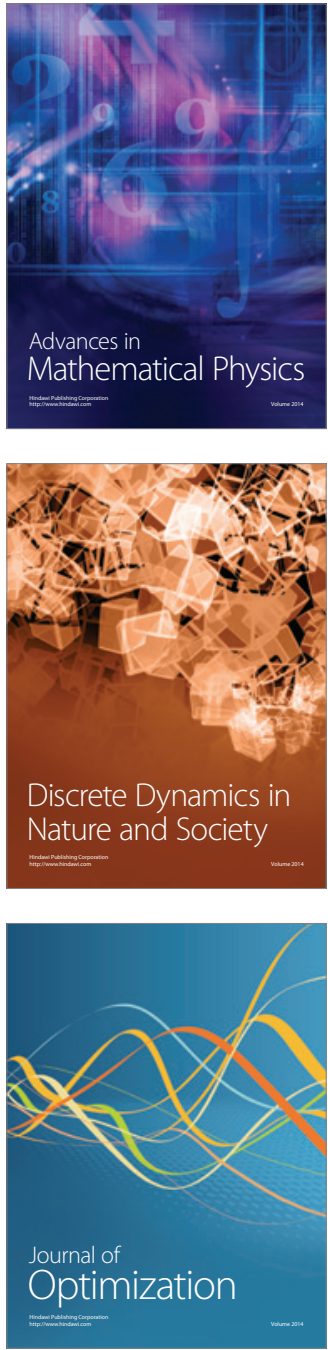